\title{
Beyond Aboutness: Classifying Causal Links in the Service of Interdisciplinarity
}

Paper prepared for the ASIST Special Interest Group on Classification Research 20th Workshop, Vancouver, November 7, 2009

\section{Claudio Gnoli, University of Pavia Rick Szostak, University of Alberta rick.szostak@ualberta.ca [contact author]}

Modern developments in all areas of knowledge tend to cross the boundaries between disciplines. ... It is very difficult to accommodate interdisciplinarity or multidisciplinarity within a traditional framework, and existing schemes are subject to increasing criticism for their failure to keep pace with changes in knowledge.

(A.C. Foskett 1996, 178)

\begin{abstract}
Most scholarship, and almost all interdisciplinary scholarship, involves the investigation of causal relationships among phenomena. Yet existing classification systems in widespread use have not focused on classifying works in terms of causal relationships. In order to allow all users interested in a particular causal link to readily find (only) all relevant works, it is necessary to develop a classification of phenomena such that each phenomenon occurs in only one place, and a classification of the sort of relationships that exist among phenomena. Such a classification would be of huge benefit to interdisciplinary scholars, and would also be useful for disciplinary scholars. In particular it will enhance the rate of discovery of 'undiscovered knowledge.'
\end{abstract}

Subject Tags: Classification, Information Retrieval, Interdisciplinarity, Knowledge Organization, Scholarly Communication

\section{Introduction}

As Bella Weinberg (1988) famously noted, library classification systems tend to classify works only with respect to what they are about, whereas scholars are interested in much more than this. The León Manifesto [<www.iskoi.org/ilc/leon.htm>] argued that interdisciplinary scholars in particular would be interested in three additional pieces of information regarding any work: the theories applied, the methods applied, and the linkages addressed (the Manifesto also calls for a non-disciplinary based classification of phenomena). This paper will focus on causal linkages - though it will be noted from time to time why classifying the theories and methods applied will also be useful.

It will be argued that coding works in terms of the causal links theorized in the work allows a (notationally simple) much greater clarity in classification, and thus renders classification much more useful to the (especially) interdisciplinary scholar. Most obviously, a researcher interested in the effects of a certain phenomenon (say a particular drug) on another (say, a particular physiological response) need not wade through the vast literature on each in order to identify works on their relationship. Less obviously, but also importantly, such a classification system can alert researchers to connections with which they may have been unaware. It thus also encourages the recognition of "undiscovered public knowledge" and therefore will significantly enhance the rate of scholarly discovery. Some existing classification systems allow causal links to be coded by agent facets and/or by influence phase relationships (Ranganathan 1967, sect. SD). However, none takes the coding of causal links as one of its major purposes (whereas the explication of causal links is the main focus of most of the scholarship we hope to classify), and all place major barriers in the way of doing so. 


\section{The Importance of Causal Links for Interdisciplinarity}

The most commonly cited definition of interdisciplinarity is as follows:

A process of answering a question, solving a problem, or addressing a topic that is too broad or complex to be dealt with adequately by a single discipline or profession ... Interdisciplinary studies draws on disciplinary perspectives and integrates their insights through construction of a more comprehensive perspective (Klein and Newell, 1996).

That is, interdisciplinarians study problems or topics that draw on the phenomena, theories, or methods of more than one discipline. While it is possible that an interdisciplinary project may focus on phenomena studied in one discipline (but applying theories or methods from others), the most common case is where interdisciplinarians study relationships between phenomena studied in different disciplines (and then also often need to confront different theoretical and methodological approaches). Interdisciplinary research, then, is usually organized around interdisciplinary causal links. The interdisciplinarian may want to know, for example, how innercity poverty is generated by a variety of economic, social, cultural, and psychological phenomena.

Existing classification systems will not leap to the aid of the interdisciplinarian. Works on inner-city poverty will be strewn across many disciplines. Even works on the relationship between culture and poverty will be classed in different places depending on whether written by an anthropologist, sociologist, economist or some other scholar. And since different disciplines tend to be organized differently in every classification (or why bother organizing classifications by discipline?), the interdisciplinarian will find that they need to use different search terms and strategies as they explore different disciplines (To be sure, verbal subject heading systems and alphabetical indexes of classifications attempt to deal with this problem, but are far from perfect).

Ideally, the interdisciplinary scholar interested in the influence of A on B would like to be able to search by:

- A, where all works about $\mathrm{A}$ are identified by a unique search term $\mathrm{A}^{*}$.

- B, where all works about B are identified by the search term B*. Since works can only be placed physically in one place, the usual prescription of "passive construction" is that works on B influenced by A be placed with other works on B. Thus an added desideratum for printed holdings would be that all works about 'determinants of B' would be physically collocated.

- The relationship from A to B. This usually has to be extracted by a Boolean search for $\mathrm{A}$ and $\mathrm{B}$. Otherwise, the search will locate huge numbers of works that address $\mathrm{A}$ in some fashion or B in some fashion but do not address how A is related to B. Note that in a library of printed documents all works on how A affects B should be filed in close proximity (though perhaps not next to each other; see next bullet). [Note here that while the focus of this paper is on causal linkages the strategies for classification advocated here could be applied as well to other sorts of relationships among phenomena such as comparisons or analogies. While less common than causal arguments, both interdisciplinary and specialized scholars often draw comparisons and analogies indeed interdisciplinarity in the humanities is particularly associated with analogy or metaphor.]

- There will often be different ways that A might affect B. These include direct causation, creation, cooperation, conflict, constraint, control, partial influence, mediation, selection, damage, and destruction (see Perreault 1965). The researcher may be able to learn much about the focus of works in this respect if these are classified in 
terms of the theories (and to a lesser extent methods) applied in a particular work. Nevertheless, it would be useful if different types of causation/influence could be distinguished. Works about B might then be collocated with respect to different types of causal influence on B.

Existing classification systems are imperfect in all these respects (Cousson 2009). Even in faceted classifications, the same phenomenon occurs in different (discipline-based) main classes, often with different notation, and often with different terminology. Works describing how A influences B are often classified under one or the other, or under both with no indication of the direction of influence. If compound notation is provided, this may also differ by class: as above a work on culture and poverty will be treated differently by discipline. Moreover, existing faceted classifications all treat causal links within a class (agent facets) quite differently from causal links across classes (influence phase relationships): this practice makes it difficult to search for a particular type of influence which might occur both within and across classes. This distinction is abolished in the freely faceted classification invoked by Austin (1976), although no such general scheme was actually produced before the recent ILC project [<www.iskoi.org/ilc/>]. Notably, the Bliss Classification (BC2) provides general rules for how links can be drawn across classes, but also provides specific instructions at many points in its schedules regarding how particular links can be made; since these deviate from the general rules, similar influence phase relationships will be treated differently depending on whether general or specific rules are to be followed.

Furthermore, its non-expressive notation cannot be exploited for automatic searches. Last but not least, existing classifications all focus the bulk of their energies on concepts expressed in terms of nouns and adjectives; there is much less development of (nominalised) verbal forms, such as "producing” or “damaging," but it is verbs that describe different types of influence.

The solutions are straightforward and point toward a freely faceted model:

- Organize a classification in terms of phenomena, not disciplines, such that each phenomenon appears only once.

- Stress the importance of causal relations by allowing any two terms to be joined by an appropriate indicator of this relationship

- Allow different types of influence to be reflected notationally.

- Then allow searches by any or all of A, B, and possible influences of A on B.

\section{A Typical Example}

Typical examples come from experience with classifying the BioAcoustic Reference Database (Gnoli et al. 2008). One representative paper in this database (Ruud and Reinen 1994) is entitled "The Effects of Car Traffic on Breeding Bird Populations in Woodland, 1: Evidence of Reduced Habitat Quality for Willow Warblers (Phylloscopus trochilus) Breeding close to a Highway”. This paper reports on a study investigating possible causal links between a technological installation (a highway producing noise) and an organic phenomenon (the size and health of a bird population). It is thus representative of a type of interdisciplinarity very common in contemporary research. Taken separately, such phenomena could be thought as the subject of completely different disciplines, engineering and biology respectively. However, the main contribution of the paper is not providing standard information about this bird species, nor about highways. Rather it is the assessment of some influence of one of them onto the other one. This is emphasized in its title by the words "Effects" and "Evidence". The latter term interestingly expresses the fact that such effects not only are discussed, but that new data are supporting the hypothesis that they actually occur. In linguistic terms, what is relevant is thus not just the 'theme' of the paper — what it is about — but its 'rheme' — what new information does it provide on the theme (Hutchins 1977; see below). This is the same difference existing in natural 
language between the participle mood ("warblers affected by highways") and the indicative mood ("warblers are affected by highways"). Experiments are being carried out in the ILC project to represent rheme notationally.

\section{What about Disciplinarians?}

Before moving on, it is worth noting that the vast bulk of disciplinary or specialized research also involves the investigation of causal links: economists worry about how changes in the money supply affect business cycles, chemists study how one chemical reacts with another to create yet another, and so on. [A minority of specialized research examines the nature or internal processes of a single phenomenon.] Causal links within disciplines are handled better by existing classification systems than causal links across disciplines, simply because each phenomenon is generally given only one place within the disciplinary main class. Yet this solves only part of the problem. Causal links are still not generally indicated as such: the researcher may thus still have to troll through a vast literature on A and B to find works on how A influences B. Even when some attempt is made to index a work in terms of relationships it is not always obvious what the work addresses: does a document indexed under "teachers", "students" and "behaviour" deals with the influence of teachers' behaviour on students, or the reverse? (Austin 1976). And different types of influence are only rarely distinguished, so again the user may retrieve many works that are not quite what they are looking for. These problems may be manageable if the researcher is interested in a small set of phenomena, and/or there are a limited number of types of influence among these that have been studied. Yet the fact remains that even disciplinary scholars will benefit from more careful treatment of causal relationships. [They may also benefit if works are coded in terms of theories and methods applied. They certainly cannot suffer from this additional information.]

How will disciplinarians fare if disciplinary structures are replaced by a phenomenon-based universal classification? Disciplinarians will benefit from being readily acquainted with research on particular linkages performed in other disciplines. They may, though, be much more interested in works generated within their own discipline. It is, of course, quite possible to continue to code works by the disciplinary home of the author. Researchers can then search only within their discipline for works on a particular causal link. This solution, though, presumes that scholars from different disciplines organize their understandings in similar ways; if not, the disciplinarian may find themselves lost in a universal classification that cannot cater to their particular way of seeing/organizing the world. Advocates of domain-specific classifications argue that we can only cope with the ambiguous nature of language by classifying works domain by domain: only then can users share an understanding of what the terms used to classify documents mean. One advantage, then, of a discipline-based general classification is that the terminology of each domain can be catered to (but with the result necessarily being that it is more difficult to search across domains). It is an empirical question as to how great the ambiguity problem is for a universal classification. If the problem is large, the ideal solution may involve domain-specific classifications, each of which is translated into a phenomenon-based universal classification (see Szostak 2010).

While the disciplinarian faces both challenges and opportunities in adapting to the sort of classification advocated by the León Manifesto, it seems likely that the opportunities will outweigh the challenges. This is especially the case once it is appreciated that the scholarly need for information science is inversely related to the degree of specialization. A very specialized scholar does not need knowledge organization. They quickly learn which journals and conferences are important. It is only as they expand their gaze beyond their narrow area of specialization that knowledge organization becomes important. If it is accepted that every scholar should have some appreciation of how their area of specialization fits within the broader scholarly enterprise, then the benefits for all scholars far outweigh the costs. 


\section{Undiscovered Public Knowledge}

Advances in human understanding come from juxtaposing previous bits of understanding that are not obviously related. Despite the centrality of relationships to knowledge organization (and advancement), existing knowledge organization systems fail to express these as usefully as they could (Green 2008, 154). We tend to evaluate information retrieval in terms of whether users find what they look for, but should instead/also appreciate the value of alerting them to a range of related paths of exploration (Warner 2000). Scholars cannot search for the unknown, but "value novelty above all else in their quests for information" (Palmer 2001, 136).

The idea of 'undiscovered public knowledge' was formally articulated in the 1960s, though the idea that there were important but unappreciated connections across distinct bodies of knowledge has been voiced since at least Aristotle. Don Swanson and others have produced many articles over the last quarter century showing how advancement in human understanding can come from drawing unappreciated connections within the existing body of knowledge (see Swanson et al 2001). Davies (1989) identifies five broad types of undiscovered knowledge: (1) hidden refutation or qualification of a hypothesis; (2) undrawn conclusions from two or more premises; (3) the cumulative evidence of weak, independent experiments; (4) solutions to analogous problems; and, (5) hidden correlations. Later Davies added a sixth category, novel classifications. Notably, Davies suggests that the amount of undiscovered public knowledge likely increases geometrically, for every new insight can potentially be combined with all preceding insights.

Davies appreciated that his problems would be greatest when connections needed to be made across disciplines. And the greatest scientific advances often involve surprising connections across diverse bodies of knowledge (Root-Bernstein 1989). Many scholars have bemoaned the difficulties faced by interdisciplinarians due to the disciplinary nature of existing classifications (e.g. Austin 1976; Beghtol 1998; Palmer 1996, 2001; Szostak 2004). Scholars within disciplines can overcome some of the limitations of document classification by talking to colleagues; interdisciplinarians will often not even know where to look or who to ask. They are thus either discouraged or forced to rely on various time-consuming and inefficient search practices: seeking out experts in other fields, tracing citations across disciplinary boundaries, searching by 'big names' in other fields, and so on.

A recent survey of the information-seeking practices of scholars found that the greatest problem is "having enough time," followed by "knowing what's available," followed by "having access to all information from one place" (see Sparks 2005). A more focused study of interdisciplinary researchers in environmental science found that 85 percent did not feel they were keeping up with all relevant research despite the fact that over a third of them were spending ten hours or more a week on information seeking activities (Murphy 2003).

Users need not master the logic behind a new system as long as they have access to a computerized search tool that is grounded in that logic (DeRidder 2007, 227). Computers cannot successfully navigate ad hoc organizing structures. They can however master a complex but logical organizing structure. Computers can then be programmed to provide users with a detailed analysis of where their search term fits within all relevant hierarchies and/or the full set of causal (or other) relationships that might be pursued.

A classification such as that outlined above should be able to help a researcher find all relevant works about the influence of A on B. They may thus be alerted to different ideas from different disciplines that when integrated provide a much better (more nuanced but also more comprehensive) understanding. This will be especially the case if they can readily distinguish different works in terms of the key arguments proposed. Researchers may often be enticed to wonder about other possible influences on B: these again would be easily searchable and the results might also lend themselves to synthesis. Or, the researcher may become intrigued by the possibility of some causal chain, and find upon investigating studies of influences on A that much 
of the observed differences in the behavior of B can in fact be traced to factors that influence A. Or the researcher may be curious about feedback effects and find unrecognized parallels between the way B influences A and A influences B. Searches by 'type of influence' would be particularly important in uncovering analogous arguments made across distinct literatures. [And searches across different types and methods would be hugely important in identifying commonalities or differences across disciplines in the evidence for similar hypotheses.] In many ways, then, the sort of classification suggested above would enhance the rate of discovery of what is at present 'undiscovered public knowledge.'

\section{Aboutness versus Relationships and Rhemes}

A work that describes how a particular drug affects a particular disease will generally be thought to be 'about' the disease. As most standard citation orders express the object acted upon before the agent, it will be classed primarily under the disease and secondarily under the drug. No mention may be made of how exactly the drug works.

However, we should want the subject entry for a work to be co-extensive with the actual subject of the work (Foskett 1996, 127). The author of the study may well feel that the most important element of the work is the explication of how the drug works. Yet this element may not be expressed in the subject classification at all. In other words, classification tends to focus on the 'theme' rather than the 'rheme' (Hutchins 1977). While novices may often wish to search by theme, scholars are likely to be as or more interested in the rheme of a work. The proposed classification approaches the issue of rhemes in a variety of ways:

- Sometimes the main claim of a work may be that A affects B in an important way. [That is, it does not just investigate possible connections but reaches a conclusion that there is a causal relationship. Only the latter is a 'rheme' within Hutchins' terminology.] Thus the simple act of coding causal links captures some rhemes. It also captures at least one element of the more common type of rheme 'A affects B in manner X.'

- Sometimes the main claim of a work may involve an assertion that theory $\mathrm{X}$ does (or does not) explain some change in B. Classifying works in terms of theories applied may thus provide some insights into the rheme of particular works.

- Likewise some works are primarily methodological in nature, the rheme being that 'Method Z allows us (or not) to investigate changes in B.' Classifying works by method applied may thus provide some access to that sort of rheme.

As useful as these approaches are, it is still useful to identify the core element of most rhemes: the claim about how exactly A affects B. This can only be achieved by classifying 'types of influence.' These can be thought of heuristically as 'verbs.' While there are important distinctions between the linguistic structure of sentences and the logical structure of information, it is nevertheless true that the rheme of most works can be approximated by a sentence of the form ' $A$ does $\mathrm{Z}$ to B.' Existing classification systems devoted the vast bulk of their attention to A and B, which for the most part are described by nouns and adjectives. It is thus necessary to investigate how verb-type relationships might be better treated in a new classification.

Just as interdisciplinarians (and disciplinarians) will benefit from a unique classification of all the phenomena involved in a causal link, they will also benefit from a general list of the types of influence involved in causal links, rather than having the same relationship treated differently in different parts of the classification. Scholars can more easily master and understand a general class of types of influence rather than a large set of overlapping and idiosyncratic verbs. More importantly, scholars may often wish to search for the same sort of influence across many different situations (as noted above). The field of communication studies in fact does just that: studies the act of communicating across a wide array of circumstances (Cesanelli 2008) (though note that in existing classifications acts of communication studied outside the field of communication studies will be classified differently from those performed by communication 
scholars). Likewise, scholars may be interested in studying 'supervising,' 'paying,' or 'conflicting with' across many distinct causal linkages.

Enumerative schemes by their nature tend to treat types of influence in an ad hoc manner. Indeed relationships are often but not always translated into phenomena in such schemes (so that 'communication' becomes a thing rather than an act). Since the BC2 unpacks each facet only within main classes, a general list of relationship is not generated, apart from that of facet categories. The Colon classification does a somewhat better job. In the (oft-ignored) seventh edition of CC, much of what had been the energy facet in the (much more often referenced and applied) sixth edition is transferred to the matter facet, leaving a set that more closely resembles types of influence (verbs). Moreover, a general list of relationships that can be used with the same notation across all main classes is generated. It includes the following verbs: create, produce, operate, infer, identify, mix, impregnate, collect data, test, design, absorb, teach, survey, review, standardize, aid, sponsor, and mediate. Yet even CC7 still maintains lengthy lists of class-specific verbs (Satija 1989, 15-6). The verb 'prevention' gets consideration under both medicine and agriculture. The education class hosts a set of verbs with more general applicability: select, administer, enroll, assess, and even accredit. The agriculture class contains some verbs that may have limited applicability elsewhere (sow, plow), but also several that clearly do (treat, cut, store, clean, sort). And even the more specific verbs might be thought of as special cases of more general verbs: sow is a way of starting a growth process, while plow is a manner of churning/mixing applied to soil.

It thus seems both feasible and desirable to develop a general set of 'types of influence' (generally verbs) that can be used across all classes. As with any other element of a library classification, we would hope to identify a small set of 'types of influence' that are used often and provide a simple notation for these. A longer list of less common verbs could then be provided with somewhat more complicated notation. Verbs that are used in only a few places might be managed with some sort of compound notation (such as using 'mix soil' for 'plow'). That approach has the added value of relating little-used verbs to a similar but more common type(s) of influence. Only detailed empirical research can establish the relative importance of different types of influence.

One verb, 'influence/cause' will surely merit special treatment. As noted above, many works focus on establishing that A influences B. They may suggest no particular type of influence or alternatively postulate myriad types of influence. [Note here that by distinguishing such works from those that posit a particular type of influence, we distinguish works that are investigative or descriptive from those that make a formal causal argument.]

A research project underway by one of the authors suggests that the following verbs are implicit across a wide variety of social science entries in DDC22:

- moving, using physical force (including threats)

- controlling/ supervising

- paying/financing

- talking/communicating to

- selecting from

- $\quad$ evaluating, judging

- $\quad$ engaging in conflicts with, between

Note that compounds of these verbs can be easily imagined: controlling plus paying could indicate controlling through financial incentives, while talking plus controlling could signal persuasion. Note also that the negative of verbs is often important: reducing conflict, not moving, being disobeyed. The vast majority of influences addressed in the social science section of DDC22 can be addressed using just this small set of verbs, their negatives, and their combinations. 
The above list of verbs captures (for the most part) causal links between phenomena. For works that focus on (at least in part) changes within a phenomenon, the following verbs are by far the most commonly invoked:

- grow or develop

- decline

- fluctuate or cycle

- exhibit stability

We have, then, some dozen verbs that can together allow us to indicate the type of influence across a huge range of social science works. It may well be that different verbs are more common in each of the humanities and natural sciences. Nevertheless, it seems likely that there is a quite manageable set of commonly used types (categories) of influence that could be indicated quite easily notationally (see Gnoli 2008). As noted above, less common verbs should also be classified, even if this requires somewhat longer (though far from excessive) notation.

\section{Implications for Classification Itself}

Most of this paper focuses on how a new classification would better serve (especially) interdisciplinary scholars. Yet it deserves to be stressed that the suggested classification likely has advantages from the perspective of knowledge organization itself. Most obviously, the new approach transcends the present practice in all existing general classifications whereby the same phenomenon is classified in multiple main classes. While this may not necessarily shorten the schedules of a classification, it will certainly not only enhance search by users but facilitate the classification of individual works: classifiers need no longer decide which disciplinary class a work falls under but can proceed to identifying the unique place in the schedules for the subject(s) of the work

The recognition of the importance of causal links also suggests a number of simple guidelines that might prove useful to both classificationist and classifier:

- Most critically, once it is appreciated that most works are best described by relationships, then compound notation should be expected to be the norm. Classificationists should thus focus on facilitating compound notation.

- Among other things, this means that we should move away from the idea inherited from the enumerative classifications developed in the nineteenth century that each work should be described by a notation that gives the impression but not the reality of a unique place in the classification. Rather, classifications should begin from the idea that a work's uniqueness reflects the particular combination of phenomena and types of influence it addresses. An expressive notation, then, would strive to make these distinct elements clear, perhaps by using facet indicators, or spaces between elements, or quite different notation (letters versus numbers versus symbols) for the different elements.

- It deserves repeating that interdisciplinary users in particular will wish to be able to search by phenomenon and type of influence. It is thus highly desirable that each phenomenon and type of influence always be treated in the same way notationally.

- The products of causal linkages will be the 'thing' that we wish to primarily classify a work under. This may seem obvious, but note that BC2 lists 'product' [of a causal relationship] as its eighth facet, thus distinguishing and distancing it from the first facet or 'thing.' A classification that devoted special attention to causal relations would not need to provide a special notation for 'results of some process.' Foskett $(1996,153)$ concurs that when a document addresses the product of some process this product should be the primary citation. Also, note that speaking in terms of phenomena and emphasizing the product of a causal relationship provides a sharper definition than Colon's Personality dimension (which Satija 1989 recognizes is hard 
to define) and avoids the complications inherent in BC2's definition of 'thing' as 'the principal foci of interest in a discipline.'

- We should want to classify most works in terms of 'what is affected,' 'how it is affected,' and 'who/what affects it.' This must be done in such a way that it is clear what is affecting what. A citation order that always listed the thing affected before the thing affecting it is important. For a causal chain, where A influences B which influences $\mathrm{C}$, these should be listed in the order $\mathrm{C}, \mathrm{B}, \mathrm{A}$ such that the intermediate role of $B$ is clear. Note, though, that this case must be distinguished notationally from a case where A and B jointly influence $C$ (In ILC the former is C6B(6A) and the latter C6B6A). There may be some advantage in a citation order of 'thing affected,' 'type of influence,' 'thing affecting.' [which may be repeated to deal with causal chains.]

\section{Concluding Remarks:}

This paper has argued that it is both important and feasible to classify scholarly works in terms of causal links. Such a classification would greatly facilitate interdisciplinary research and especially the discovery of undiscovered public knowledge. It is also likely to enhance more specialized research. Last but not least, it suggests ways in which both the tasks of classification and of classifying particular works can be improved.

\section{References:}

Austin, Derek (1976), “The CRG Research into a Freely Faceted Scheme” in Arthur Maltby, ed., Classification in the 1970s: A Second Look. London: Bingley, p. 158-194.

Beghtol, Clare (1998), "Knowledge Domains: Multidisciplinarity and Bibliographic Classification Systems”, Knowledge Organization, 25:1-2, 1-12.

Cesanelli, Enzo (2008), "Classificare il dominio della comunicazione secondo la teoria dei livelli di integrazione”, in E-LIS, <http://eprints.rclis.org/14632/>.

Cousson, Philippe (2009), "UDC as a non-disciplinary classification system for a high-school library", in Proceedings of Classification at a crossroads, The Hague, 29-30 October 2009, Extensions and Corrections to the UDC, in press.

Davies, Ron (1989) "The Creation of New Knowledge by Information Retrieval and Classification” Journal of Documentation 45:4, 273-301.

DeRidder, Jody L. (2007) "The Immediate Prospects for the Application of Ontologies in Digital Libraries,” Knowledge Organization 34:4, 227-46.

Foskett, A.C. (1996) The Subject Approach to Information. $5^{\text {th }}$ edition. London: Library Association Publishing.

Gnoli, Claudio (2006) The Meaning of Facets in Nondisciplinary Classifications, in Knowledge Organization for a Global Learning Society: Proceedings, 9th ISKO Conference, Vienna, 4-7 July 2006, G. Budin et al. eds., Würzburg: Ergon, 2006, p. 11-18, $<$ http://www.iskoi.org/ilc/facets.proc.rtf $>$.

Gnoli, Claudio (2008) Categories and Facets for Integrative Levels. Axiomathes, 18:2, 177-192.

Gnoli, Claudio, Gabriele Merli, Gianni Pavan, Elisabetta Bernuzzi, and Marco Priano (2008) Freely Faceted Classification for a Web-based Bibliographic Archive: the BioAcoustic Reference Database, in Repositories of knowledge in digital spaces: proceedings Eleventh German ISKO Conference, Konstanz, 20-22 February 2008, Würzburg: Ergon. Also in “DLIST", <http://dlist.sir.arizona.edu/2274/>.

Green, Rebecca (2008) "Relationships in Knowledge Organization,” Knowledge Organization $35: 2 / 3,150-9$.

Hutchins, W. John (1977) On the Problem of “Aboutness” in Document Analysis. Journal of Informatics, 1, 17-35. 
Klein, Julie Thompson, and William H. Newell (1996) “Advancing Interdisciplinary Studies” in Jerry G. Gaff, James Ratcliff, and Associates, eds., Handbook of the Undergraduate Curriculum. San Francisco: Jossey-Bass.

Murphy, Janet (2003) “Information-Seeking Habits of Environmental Scientists” Issues in Science and Technology Librarianship 38 (Summer).

Palmer, Carole L. (1996) "Information work at the boundaries of science: linking library services to research practices” Library Trends, 45:2, 165-191.

Perreault, J.M. (1965) "Categories and relators: a new schema”, Revue internationale de documentation, 32, 136-144. Republished in International Classification, 21:4, 189-198.

Ranganathan, S.R. (1967) Prolegomena to Library Classification, 3rd ed. Bangalore: SRELS. Also in "DLIST", <http://dlist.sir.arizona.edu/1151/>.

Reijnen, Rien and Ruud Foppen (1994) "The Effects of Car Traffic on Breeding Bird Populations in Woodland, 1: Evidence of Reduced Habitat Quality for Willow Warblers (Phylloscopus trochilus) Breeding close to a Highway.” Journal of Applied Ecology 31, 85-94.

Root-Bernstein, Robert (1989) Discovery. Cambridge: Harvard University Press.

Swanson, Don R., Neil R. Smalheiser, and A. Bookstein (2001) "Information Discovery from Complementary Literatures: Categorizing Viruses as Potential Weapons." Journal of the American Society for Information Science and Technology 52, 797-812.

Szostak, Rick (2004) Classifying Science: Phenomena, Data, Theory, Method, Practice. (Dordrecht: Springer).

Szostak, Rick (2008) Classification, Interdisciplinarity, and the Study of Science. Journal of Documentation, 64:3, 319-32.

Szostak, Rick (2010) "Universal and Domain-specific Classifications from an Interdisciplinary Perspective” proceedings of the 11th international ISKO conference, Rome, 2010, Würzburg: Ergon.

Szostak, Rick, and Claudio Gnoli (2008) "Classifying by phenomena, theories, and methods: examples with focused social science theories," in Culture and identity in knowledge organization, proceedings of the 10th international ISKO conference, Montréal, 2008, Würzburg: Ergon, p. 205-211.

Weinberg, Bella Hass (1988) "Why indexing fails the researcher” The Indexer 16:1, 3-6. 